\title{
1 Hymenoptera associated eukaryotic virome lacks host
}

\section{2 specificity}

3

4 Ward Deboutte* ${ }_{1}$, Leen Beller1, Claude Kwe Yinda1,2, Chenyan Shi1, Lena Smets1,

5 Bert Vanmechelen1, Nadia Conceição-Neto1, Kai Dallmeier3, Piet Maes1, Dirk C de

6 Graaf4, Jelle Matthijnssens* ${ }_{1}$

\section{Author Affiliations}

$8{ }_{1}$ KU Leuven - University of Leuven, Department of Microbiology, Immunology and Transplantation,

9 Rega Institute for Medical Research, Division of Clinical and Epidemiological Virology, 3000, Leuven,

10 Belgium

112 NIAID/NIH, Rocky Mountain Laboratories, Laboratory of Virology, Virus Ecology Unit,59840, Montana,

12 USA

13 з KU Leuven - University of Leuven, Department of Microbiology, Immunology and Transplantation,

14 Rega Institute for Medical Research, Laboratory of Virology and Chemotherapy, 3000 Leuven, Belgium

154 UGent - Ghent University, Department of Biochemistry and Microbiology, Laboratory of Molecular

16 Entomology and Bee Pathology (L-MEB),9000, Ghent, Belgium

17

*Correspondence: ward.deboutte@kuleuven.be; jelle.matthijnssens@kuleuven.be 


\section{Abstract}

19 Recent advancements in sequencing technologies and metagenomic studies have

20 increased the knowledge of the virosphere associated with honey bees tremendously.

21 In this study, viral-like particle enrichment and deep sequencing was deployed to detect viral communities in managed Belgian honey bees. A substantial number of previously undescribed divergent virus genomes was detected, including a rhabdovirus and a recombinant virus possessing a divergent Lake Sinai Virus capsid and a Hepe-like polymerase. Furthermore, screening $>5,000$ public sequencing datasets for the retrieved set of viral genomes revealed an additional plethora of undetected, divergent viruses present in a wide range of Hymenoptera species. The unexpected high number of shared viral genomes within the Apidae family and across different families within the order Hymenoptera suggests that many of these viruses are highly promiscuous, that virus sharing within and between Hymenoptera families occurs frequently, and that the concept of species-specific viral taxa inside the Hymenoptera should be revisited. In particular, this estimation implies that sharing of several viral species, thought to be specific for bees, across other eukaryotic taxa is rampant. This study provides important insights on the host taxonomical breadth of some of the known "bee viruses" and might have important implications on strategies to combat viruses that are relevant to pollinators.

38 Introduction

39 The European honey bee (Apis Mellifera) forms a central hub in ecosystem

40 maintenance, resilience and diversity. Aside from the economically valuable products, 41 such as honey and nectar $(1,2)$, managed bee colonies together with other insects 
contribute tremendously to pollination (3) and play a key role in global agricultural production (4). In the past decades, pressures on both managed and wild bees have increased vastly and there is evidence for declining trends in pollinator populations globally $(5,6)$. These pressures encompass ecological factors such as habitat loss $(7)$, pollution (8), pesticide use $(9,10)$ and adverse agricultural practices $(11)$, but biological factors including bacterial, parasitic, and viral infections (12-15), also play a pivotal role. Recently, more attention is being given to the microbiota and their influence on bee health, development and homeostasis (16-18), and it has been shown that the microbiota can be exploited to protect bees from other pathogens (19). The influence these factors have can be cumulative or even synergistic. For example, it has been shown that pesticide use can perturb the expression of essential immunocompetence genes, increasing the probability of microbial infections (20). Perhaps the best example for mutual synergistic factors detrimental for bee health confine parasitic and viral infections. The worldwide spread of the Varroa destructor parasite facilitated Deformed wing virus (DWV) infections by acting as an active vector (where the virus can replicate in both the vector and the host) (21). Parallel to its role as viral vector, it has been shown that the $V$. destructor parasite can also influence the immune status of its host (22). Globalization of $V$. destructor and concomitant DWV infections raised the question what influence DWV plays in colony health. Recent studies have revealed an association between DWV infections and colony health status (23-25). Despite the worldwide dominance of DWV, other RNA viruses have been shown to be highly virulent, resulting in a strong phenotype in infected bees. Acute bee paralysis virus (ABPV), Black queen cell virus (BQCV) and Sacbrood virus (SBV) are all members of the order Picornavirales that have a detrimental effect on colony health once they infect a hive (26). Scattered information suggests that some of these viruses are not 
67 to be restricted to honey bees, but also infect and replicate in other members of the Apidae family. Spill-over events from managed honey bees into bumblebee species have been described for DWV, BQCV, ABPV, SBV and Lake Sinai viruses (LSV) (2730), whereas honey bee viruses have also been described in ants (Formicidae) (31) and wasps (Vespidae) (32). Recent advancements in sequencing technologies and metagenomics have accelerated virus discovery in bees and a number of studies have attempted to describe the viral diversity associated with bees. These studies were able to expand the range of known honey bee viruses significantly and aside from numerous viruses belonging to the order Picornavirales, numerous other RNA viruses have been discovered belonging to the orders Bunyavirales, Mononegavirales 77 (containing the family Rhabdoviridae) and Articulavirales (containing the family Orthomyxoviridae), and several unclassified RNA viruses such as LSV (33-37). DNA viruses have also been described, such as Apis mellifera Filamentous virus (AmFV) (38), and numerous single-stranded DNA viruses (39). While these sequencing efforts have vastly increased the number of known honey bee related viruses, the relevance of most of these viruses remains enigmatic. In this study, we first describe the eukaryotic viruses present in $>300$ Belgian bee colonies collected in the framework of the EpiloBEE study (40) in 2012 and 2013. We place these results in the context of other known insect viruses. Finally, by screening more than 5,000 public RNA sequencing datasets, we shed light on the sharing of (bee) viruses between different members of the order Hymenoptera and within the Apidae lineage. 


\section{Results}

89 Eukaryotic virus identification yields previously known and unknown honey bee viruses

91 Viral-like particle enrichment (41) and Illumina sequencing was performed on pooled samples derived from 300 weak and healthy (as defined by the EpiloBEE study (40)) managed honey bee colonies in Flanders, Belgium as described before (42). After sequencing and de novo assembly of the individual libraries, redundancy of the retrieved contigs was removed by collapsing sequences with $97 \%$ nucleotide identity over $80 \%$ of their length. Subsequently, the non-redundant contig set was annotated using DIAMOND (43) against NCBI's NR database. Viruses were taxonomically classified using the lowest-common ancestor algorithm implemented in Kronatools (44). Sequences showing similarity to bacteriophages were omitted from this analysis.

100 Genome coverage values were obtained by mapping the sequencing reads per sample back to the non-redundant contig set. Clustering analysis on the viral coverage matrix revealed a distinct clustering pattern between samples derived from weak and healthy colonies, although with a very small biological relevance (adonis test, $\mathrm{R}^{2}=$ 0.035, $p$-value $=0.0042)($ Fig. $1 \mathrm{~A})$. The log-transformed coverage matrix showed that the vast majority of viral reads could be attributed to the family Iflaviridae, of which DWV is a member (Fig. 1B). The second most prevalent viral family was the family Orthomyxoviridae. Several families containing plant and fungal viruses, such as

108 Partitiviridae, Chrysoviridae, and Tymoviridae, were also recovered. The clustering 109 pattern of the coverage matrix reflected the adonis test results, showing most of the samples being dispersed by health status and although one healthy cluster containing

111 mainly unclassified reads exists, the lack of monophyly implies no clear differences in 112 composition with respect to the health status. In terms of absolute contig count, the 
113 most prevalent orders were (apart from unclassified sequences) Picornavirales,

114 Tymovirales and Mononegavirales (supplemental fig. S1A) and the most prevalent

115 families were (next to unclassified sequences) Partitiviridae, Comoviridae, and

116 Parvoviridae (supplemental fig. S1B). There was no significant difference between the

117 number of non-redundant contigs present in healthy and weak samples (Mann-

118 Whitney $U$ test, p-value $=0.32)$ (supplemental fig. S2). Only $30 \%$ of the non-redundant

119 contigs had an amino acid similarity percentage with the best hit in the NR database

120 higher than $90 \%$, reflecting the divergent nature of the retrieved sequences

121 (supplemental fig. S3). Species accumulation curves revealed a near horizontal

122 asymptote, implying that viral sequence space was probed sufficiently (supplemental

123 fig. S4). The relatively short length of the majority of retrieved viral sequences

124 hampered a complete phylogenetic analysis (supplemental fig. S3). Therefore, an allby-all TBlastX search was conducted using the retrieved non-redundant contig set

126 complemented with a filtered viral Refseq set (see methods) as both query and bait.

127 The resulting blast output was converted into a network using sequences as vertices, and hits as edges. A minimized-nested block network was constructed and visualized using the taxonomical information of the reference sequences (Fig. 1C). The vast majority of retrieved sequences clustered together in blocks with the order

131 Picornavirales, although the orders Bunyavirales, Mononegavirales and Tymovirales

132 were also represented substantially. Several contigs could not be assigned to any

133 known order and represented unclassified (ds)RNA viruses or unclassified (circular)

134 DNA viruses. 

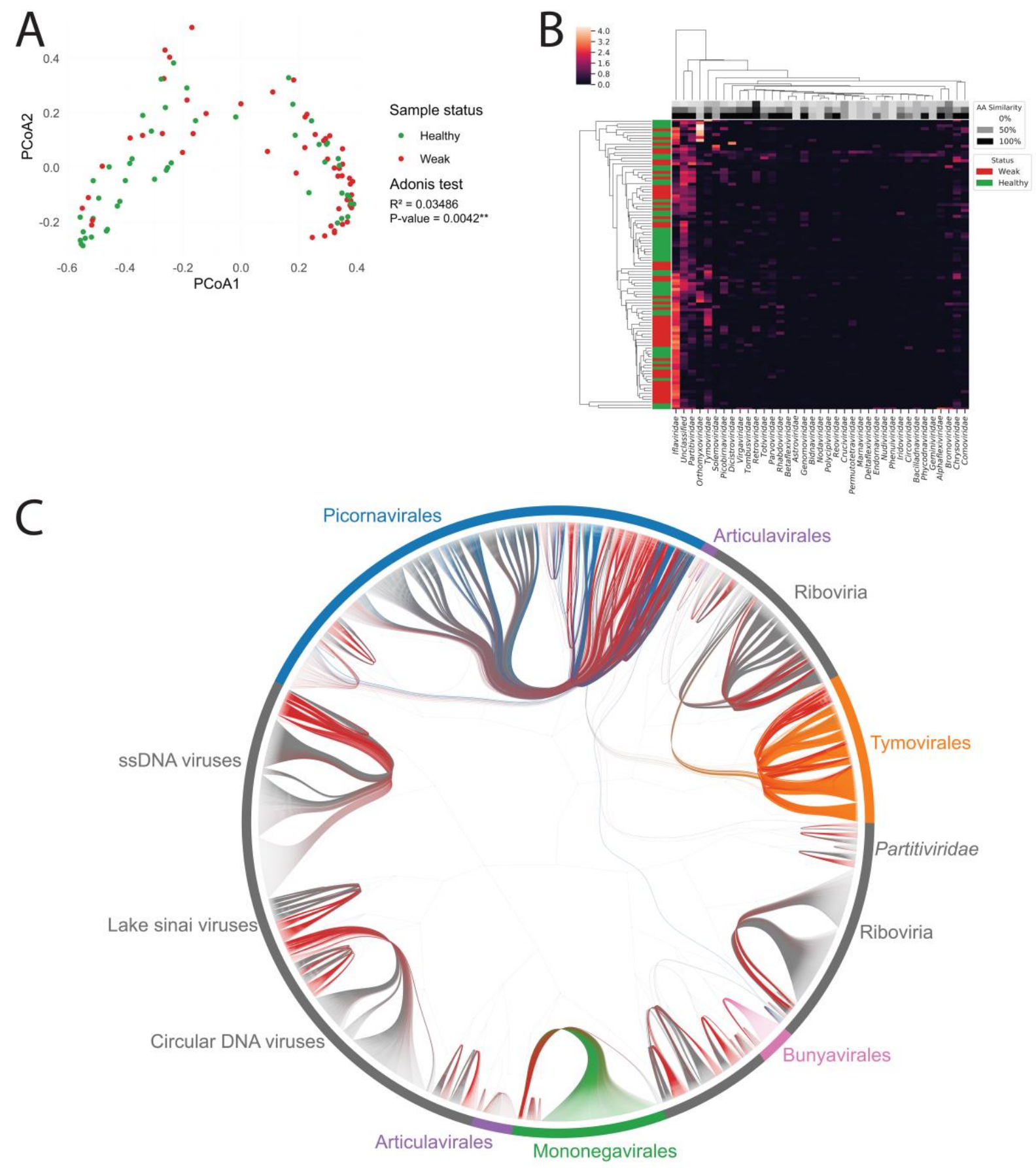

136 Fig. 1. Belgian honey bees harbor a diverse range of known and novel viruses.

137 (A) PCoA clustering using Bray-Curtis distances calculated on the viral coverage matrix derived from 138 the Belgian samples $(n=102)$. Green dots reflect samples derived from healthy colonies; red dots reflect samples derived from weak colonies. The $R$ squared and $p$-value obtained from the Adonis test are indicated on the right. (B) Average values per viral family of the log-transformed viral coverage matrix are depicted in a heatmap, clustered using Euclidian distances. The left column depicts samples derived from healthy (green) and weak (red) samples. The first three rows indicate the minimum (top), the average (middle) and the maximum (bottom) percentage of amino acid similarity of the contigs per viral family. (C) Minimized nested block network using retrieved sequences in this study (red) and known Refseq viruses (all other colors). Known orders are indicated in colors and unclassified reference sequences are indicated in gray. 
147 Phylogenetic analysis confirms the presence of known and divergent

\section{eukaryotic viruses in Belgium}

149 To investigate the phylogenetic placement of a subset of the retrieved near-complete

150 viral genomes, maximum clade credibility trees (MCC) were created using BEAST (45)

151 (Fig. 2). Retrieved genomes from this study (orange tip labels, and listed in

supplemental table 1) and from the short-read sequencing archive (SRA, NCBI) search (blue tip labels, see below), as well as reference sequences (green tips), were included based on sequence length and based on a BlastP search (see methods). Phylogenies were created for Rhabdo-like, Picorna-like, Bunya-like, Orthomyxo-like,

156 Sinai-like, Partiti-like, Toti-like and Tymo-like viruses. One of the retrieved rhabdo-like

157 viruses (Apis rhabdovirus1-Belgium) was nearly identical to the recently identified Apis rhabdovirus 1 (34), while the other Rhabdo-like virus (Apis rhabdovirus3-Belgium) has

Diachasmimorpha longicaudata rhabdovirus as closest relative (but only had $38 \%$ amino acid identity for the $L$ protein). The retrieved Picorna-like viruses reflect known bee pathogens clading in the families Iflaviridae and Dicistroviridae, such as DWV, SBV and ABPV, but also include more divergent sequences related to Nora-like viruses. A number of sequences clading together with plant infecting picornaviruses, such as several comoviruses were also retrieved. The retrieved Orthomyxo-like viruses are three closely related viruses (Apis orthomyxovirus 1, 2 and 3-Belgium),

166 clustering together with other known thogotoviruses. These three viruses are nearly 167 identical to the recently discovered Varroa Orthomyxovirus, with the exception of the nucleoprotein (35). Furthermore, five LSV-like viruses were retrieved, out of which four were very similar to other known Lake Sinai viruses (between 94\% and 97\% nucleotide similarity). Interestingly, the fifth identified Lake Sinai virus was initially identified as an 
bioRxiv preprint doi: https://doi.org/10.1101/2020.09.15.298042; this version posted September 16, 2020. The copyright holder for this preprint (which was not certified by peer review) is the author/funder, who has granted bioRxiv a license to display the preprint in perpetuity. It is made available under aCC-BY-NC-ND 4.0 International license.

173 Bastrovirus-like virus), and a Lake Sinai virus-like capsid (Lake Sinai virus, 35\% amino

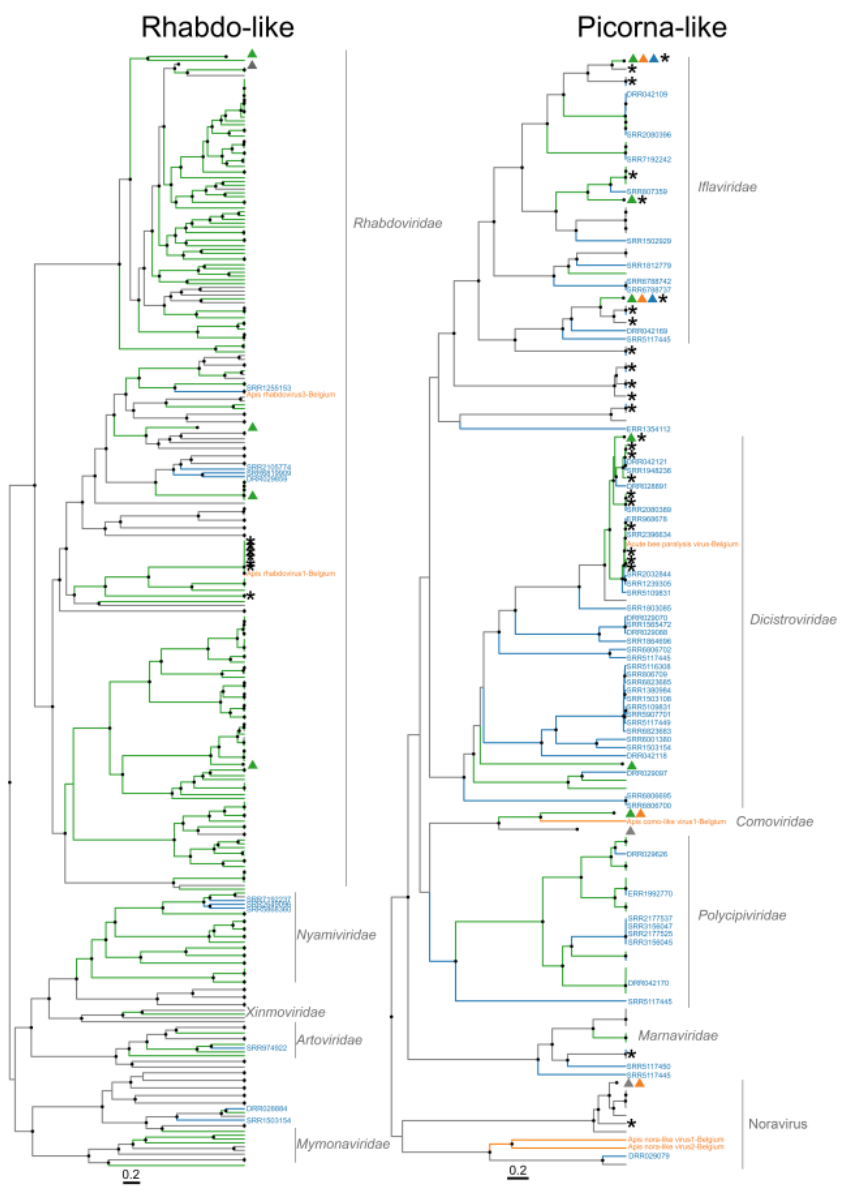

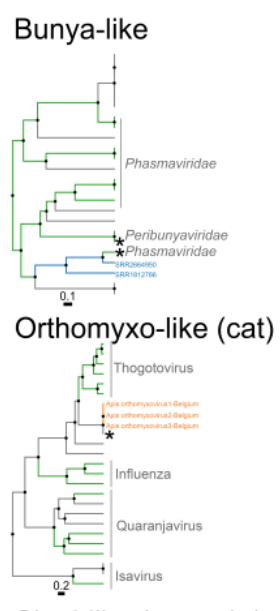

Sinai-like (recomb.)

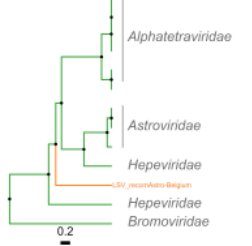

Partiti-like

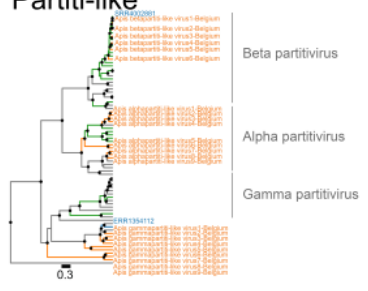

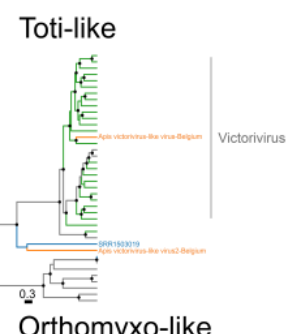

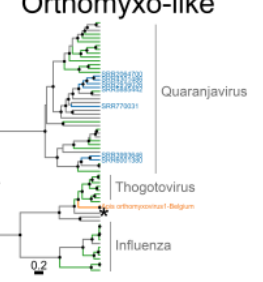

Sinai-like*
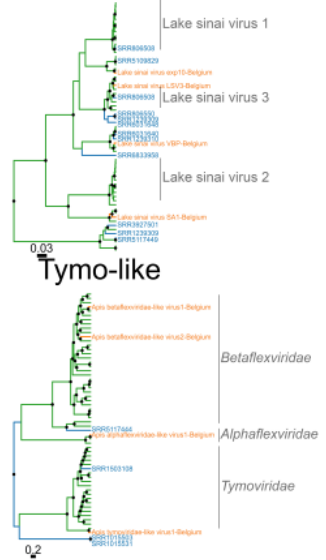

Fig. 2. Phylogenetic analysis highlights the vast diversity of viruses identified in the Belgian samples.

Maximum clade credibility trees for the best-represented groups of viruses retrieved in this study. Black circles on the nodes indicate posterior support values $>0.9$. Viruses identified in the Belgian samples are indicated with orange tip labels, those identified through SRA searches are indicated with blue tip labels. Reference sequences that belong to a classified viral family or genus are indicated with green branches. Known honey bee viruses are indicated with an asterisk. One or more triangles indicate collapsed clades, and the colors are equivalent to the tip and branch colors. Clades that belong to the same family or genus are indicated with a gray line and in text. The 'Rhabdo-like' tree is built using the putative $L$ protein. The 'Picorna-like' tree is built using the putative polyprotein (monocistronic viruses), the putative ORF1 (dicistronic viruses) or the putative replication polyprotein (Nora-like viruses). The

191 'Bunya-like' tree is built using the putative $L$ protein. The 'Toti-like' tree and the 'Partiti-like' tree are built 
192 using the putative RdRP gene. The 'Orthomyxo-like (cat)' tree is built using a concatenated protein

193 alignment of the putative PB2 - PB1 - PA - NP genes, while the 'Orthomyxo-like' tree is built using

194 only the PB2 segment. The 'Sinai-like (recomb.)' tree is built using the putative polymerase gene of the

195 astrovirus-LSV recombinant virus, while the 'Sinai-like' tree is built using the putative polymerase region of all the known LSV viruses (not including the recombinant). The 'Tymo-like' tree is built using the putative polyprotein gene.

Re-screening of existing RNA sequencing datasets reveals untapped viral

201 Since the recovered viral sequences included most of the known honey bee viral sequence space (Fig. 1C), the assumption was made that the non-redundant viral dataset we recovered was a good reflection of all known honey bee viruses. This dataset was used as bait to map a total of 5,246 RNA sequencing datasets found in the SRA database when using the query 'Hymenoptera + RNA'. A dataset was considered to be 'virus enriched' when at least 100,000 reads mapped to the bait set. All datasets that met this criterium $(1,331)$ were individually de novo assembled using SKESA (46) and viral sequences were identified and clustered as was described for the Belgian samples. An additional clustering step was performed, collapsing the nonredundant SRA-derived sequences together with the non-redundant Belgian

211 sequence dataset. This resulted in the recovery of nearly 10,000 non-redundant 212 putative viral contigs, out of which only $42.8 \%$ had an amino acid similarity with proteins in Genbank higher than 90\% (supplemental fig. S6). Forward model selection analysis revealed that together, putative host taxonomy and location of the dataset could explain $33 \%$ of the variability observed within the coverage matrix (Fig. 3A). This

216 result was further validated by the observation that hierarchical clustering on Euclidian

217 distances revealed clusters of both eukaryotic host families and location within the 218 coverage matrix (Fig. 3B). Viral taxonomy analysis revealed that the majority of the 219 recovered viruses could be assigned to the orders Picornavirales and 220 Mononegavirales (Fig. 3C). The retrieved viral contigs that fell below the 
221 abovementioned threshold of $90 \%$ amino acid similarity were included in the

222 phylogenetic analysis and revealed ten previously undescribed Rhabdo-like viruses,

223 and more than 50 previously undescribed Picorna-like viruses (Fig. 2, blue tip labels).

224 Both these groups span multiple viral families. Another striking finding was the fact

225 that seven previously undescribed PB2 segments of Orthomyxoviridae-like sequences

226 were recovered (most closely related to the Quaranjavirus genus), indicating that this

227 viral family is more strongly represented within the Hymenoptera lineage than was

228 previously known. Furthermore, also Bunya-like, Toti-like, Sinai-like, Partiti-like and

229 Tymo-like viruses were recovered (Fig. 2, blue tip labels). 

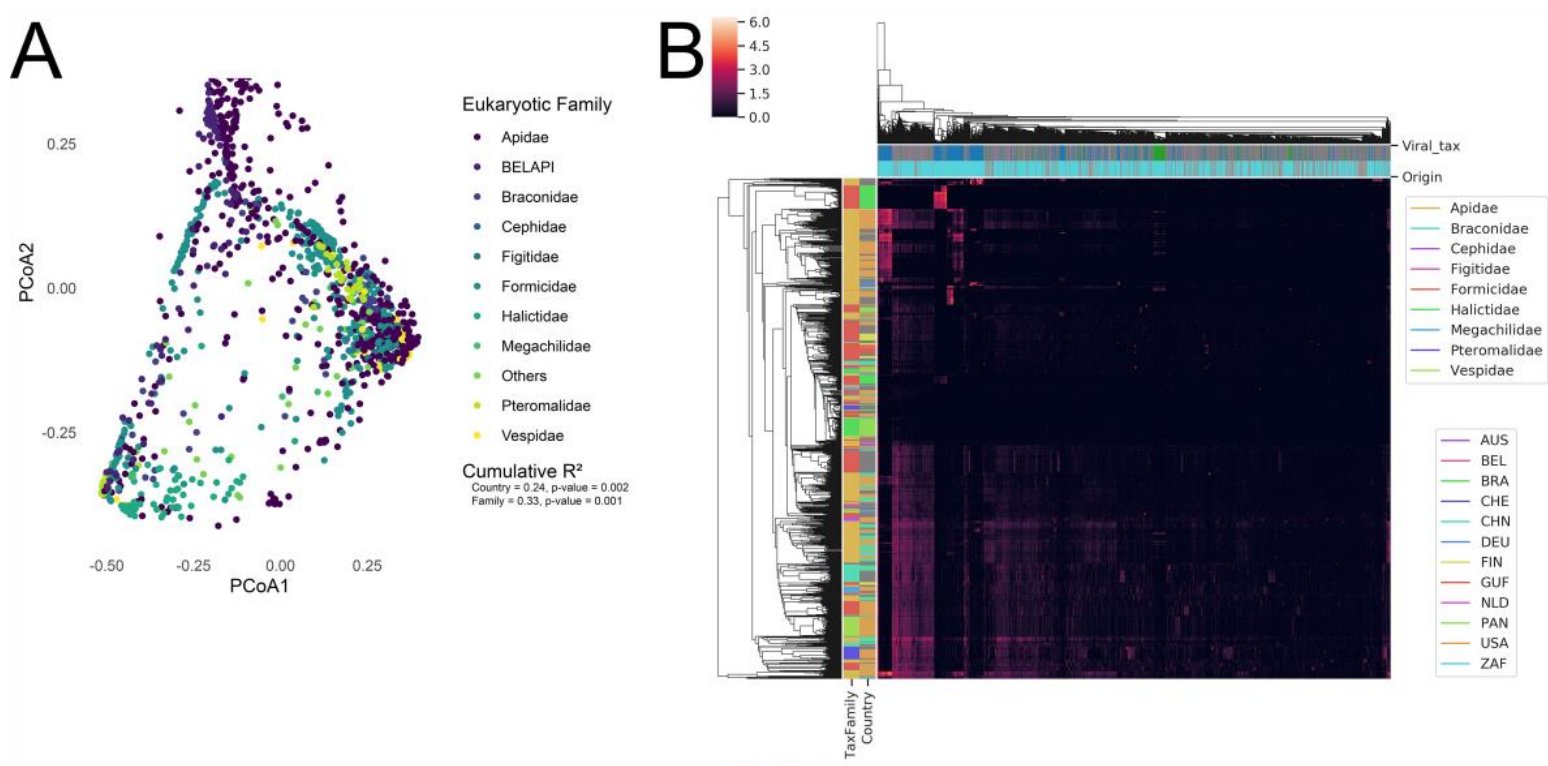

C

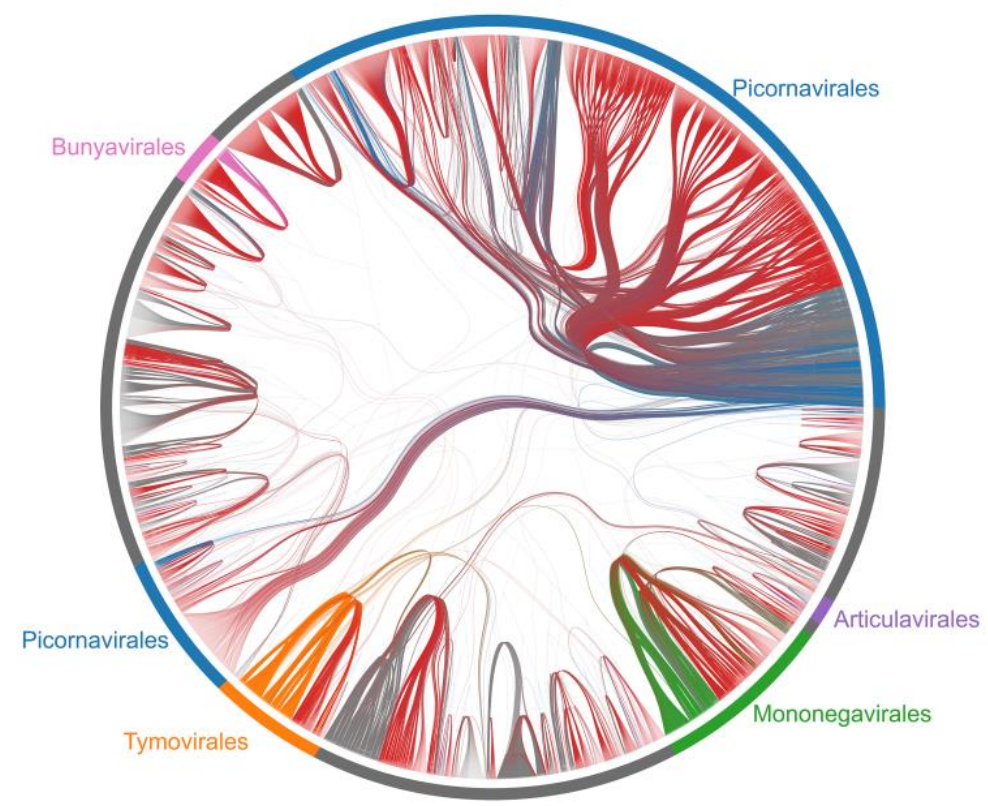

Fig. 3. SRA searches shed light on the hymenoptera virosphere and reveal the wealth of undescribed viral sequences present in public datasets.

(A) PCoA clustering using Bray-Curtis distances calculated on the viral coverage matrix derived from the Belgian samples clustered with the SRA screening results. Dots are colored per hymenoptera family. The cumulative $R_{2}$ values reported are calculated by forward model selection using the OrdiR2step function after distance-based redundancy analysis. (B) Heatmap depiction of the log transformed viral coverage matrix, clustered using euclidian distances. Leftmost columns indicate the hymenoptera families and the geographical location of the samples. The top two rows indicate the viral taxonomical classification (with the same color per viral order as Fig. $3 \mathrm{C}$ ) and the origin of the viral sequence (light blue indicates an SRA sample as origin, red indicates viral sequences found in the Belgian samples). (C) Minimized nested block network using the non-redundant sequences retrieved from the SRA searches (red) and known Refseq viruses (all other colors). 
244 Virus-sharing networks show a large number of virus sharing within the order

\section{of Hymenoptera}

246 Although the discrimination between samples based on their location and eukaryotic

247 taxonomy was significant (p-value 0.002 and 0.001 , respectively) (Fig. 3A). The

248 cumulative $\mathrm{R}_{2}$ value (0.33) indicates that a large majority of the variances within the

249 datasets cannot be explained by aforementioned variables. This observation could

250 imply that a large number of viruses are shared across hymenoptera families, that the

251 variance within eukaryotic families in a specific country is large relative to the variance

between these parameters, or a combination of both. To investigate the first possibility,

the assumption was made that the host of a specific virus sequence was that of the

sample of which the sequence cluster representative was derived. Hymenoptera

families of which less than ten samples were obtained were grouped together into an

'Others' group and virus sharing was calculated in a pair-wise manner for all the

possible combinations within the eukaryotic host families, and within the Apidae

lineage. A substantial number of viruses were found to be present not only within the

Apidae lineage but also shared over multiple eukaryotic host families (Fig. 4 A, B).

Within the family Apidae, most viral sequences were shared between Apis Mellifera Apis florea (938 sequences shared), and between Apis Mellifera and Ceratina species

(729 sequences shared) (Fig. 4A). The majority of these shared sequences could be traced back to the order Picornavirales, with a total of $224(21.3 \%), 497(53.0 \%)$ and 130 Picorna-like sequences (17.8\%) shared between these groups, respectively (Fig. between the families Apidae and Pteromalidae (1,066 viral sequenced shared), the 
269 Braconidae (737 sequences shared). Concomitant with the situation between different

270 Apidae species, the majority of shared viral sequences could be assigned to the order

271 Picornavirales, with 201 (18.8\%), 137 (18.4\%) and 111 Picorna-like sequences

272 (15.0\%) shared between these groups, respectively (Fig. 4D, blue edges). Aside from

273 Picorna-like sequences, evidence could also be found for sharing of viruses predicted

274 to belong to the orders Mononegavirales (Fig. 4C,D, green edges) and Tymovirales

275 (Fig. 4C,D, orange lines), although the number of shared viral sequences was on general an order of magnitude lower than those of the Picornavirales (39 Mononega-

277 like viral sequences shared between Formicidae and Pteromalidae, and 27 Tymo-like

278 viral sequences shared between Apis Mellifera and Lepidotrogona). Since a fraction

279 of the recovered viruses are most likely infecting plants or reflect viruses not relevant

280 for bees (Fig. 2), an additional analysis was ran with a number of the retrieved, nearcomplete, known bee viruses (AMFV, ABPV, BQCV, Kashmir Bee virus (KBV), DWV,

LSV, Apis Rhabdovirus and Apis Orthomyxovirus), as well as the retrieved Nora-like viruses and other Orthomyxo-like viruses. Calculation of the fraction of positive samples revealed that most of the previously thought bee-specific viruses occur in multiple Apidae species but are also found within other Hymenopteran families (supplemental fig. S7). An attempt was made to quantify the host specificity of these viruses by calculating an Apidae specificity index (ASI), and an Apis Mellifera specificity index (AMSI) (Table 1). These indices revealed that some of the established bee viruses (ABPV, AMFV, BQCV and Quaranja-like orthomyxoviruses) show a low specificity for Apis Mellifera within the Apidae family (characterized by a low AMSI), and (with the exception of BQCV) were not restricted within the family Apidae

292 (characterized by a low ASI). Other "established honey bee species" were shown to 293 be highly specific for Apis Mellifera, and revealed a high AMSI (KBV, DWV and LSV). 
294 The recently discovered Apis rhabdoviruses and Nora-like viruses are found 295 exclusively in Apis Mellifera within the family Apidae. The Apis rhabdoviruses are

296 restricted within the family Apidae, but the retrieved Nora-like viruses are also highly 297 prevalent in other Hymenoptera families (ASI 0.01, Table 1). Finally, the retrieved 298 Quaranja orthomyxo-like viruses were highly prevalent in other Hymenoptera families 299 and only to a limited extent in Apis Mellifera and the family Apidae (ASI of 0.04 and 300 AMSI of 0.05 , respectively). On the other hand, Apis Orthomyxovirus 1 was slightly 301 more honey bee specific, with an ASI and AMSI of 0.31 and 0.21 , respectively. 
A

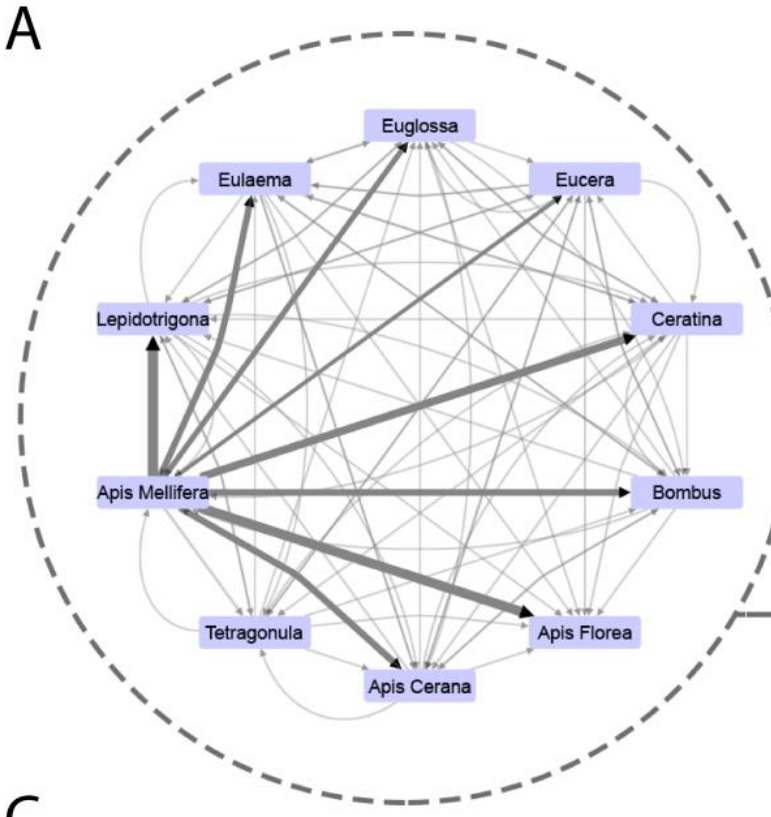

C

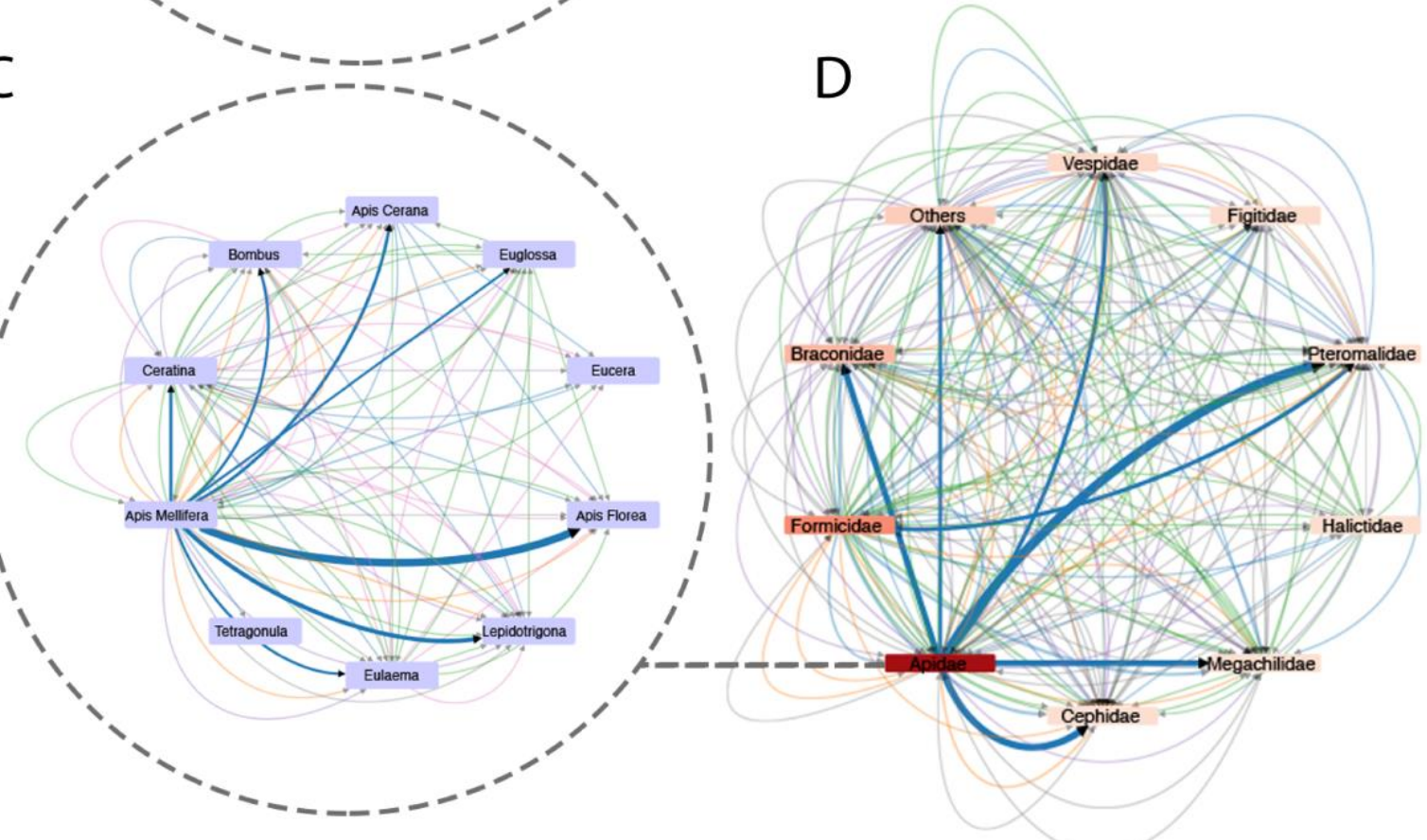

B

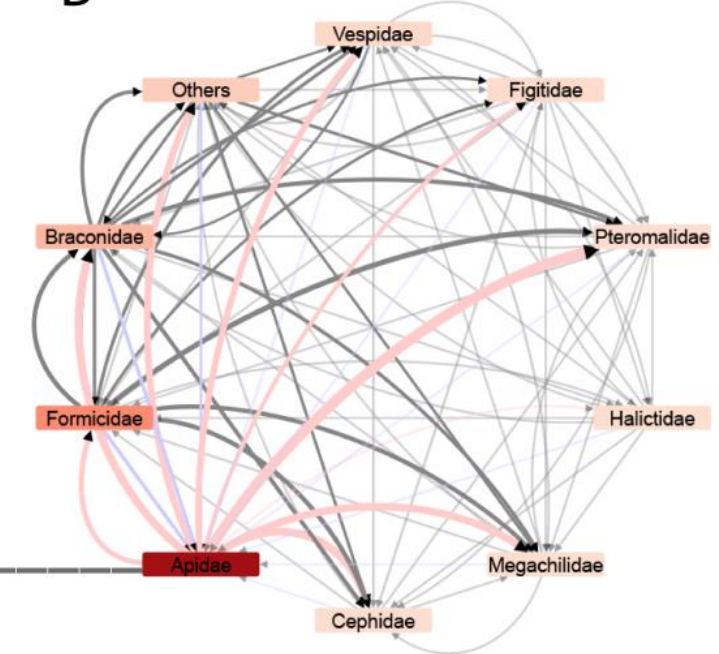

Fig. 4. Cumulative viral sequence sharing network reflect the aspecificity of hymenoptera associated viruses.

Networks reflecting the cumulative sharing of viral contigs between eukaryotic lineages. The networks inside the dashed circle $(A, C)$ reflect viral sequence sharing within the family Apidae. The networks on the right $(B, D)$ reflect sharing over different families within the order hymenoptera. Panels $A$ and $C$ and panels $B$ and $D$ both reflect the same networks, but both panels $C$ and $D$ reflect cumulative shared viral sequences broken up per assigned viral order (using the same color code as fig. 3C). Nodes in panels $B$ and $D$ are colored by number of representative virus contigs per eukaryotic lineage (ranging from 18 contigs (Cephidae) to 5,662 contigs (Apidae)). Edge thickness reflects the total shared contig count, ranging from 1 to 1,050 contigs (panel A), from 1 to 1,066 contigs (panel B), from 1 to 497 contigs (panel

313 C), and from 1 to 201 contigs (panel D). Edge arrows indicate directionality, of which the root is the predicted host (the taxonomical group of which the virus sequence representative was derived from). 
Table 1. Host (a)specificity of a selection of known bee viruses.

318 The values reflecting how specific a known bee virus is for Apis mellifera (AMSI) and for Apidae (ASI).

319 A value of 1 reflects complete lineage restriction. The number of viral contigs included per viral species

320 is indicated with Contig number.

321

\begin{tabular}{lllll}
\hline Virus & Virus Abbreviation & Contig number & ASI & AMSI \\
\hline Acute Bee Paralysis virus & ABPV & 7 & 0.13 & 0.04 \\
\hline Apis Mellifera Filamentous virus & AMFV & 8 & 0.06 & 0.05 \\
\hline Kashmir Bee virus & KBV & 2 & 0.01 & 1.00 \\
\hline Deformed Wing virus & DWV & 11 & 0.84 & 0.71 \\
\hline Black Queen Cell virus & BQCV & 7 & 0.88 & 0.09 \\
\hline Lake Sinai viruses & Sinaiviruses & 20 & 0.07 & 1.00 \\
\hline Apis Rhabdovirus (1 and 2) & Rhabdo & 4 & 1.00 & 1.00 \\
\hline Quaranja-like Orthomyxoviruses & Quaranja & 15 & 0.05 & 0.04 \\
\hline Apis Orthomyxovirus 1 & Thogoto & 3 & 0.31 & 0.21 \\
\hline Nora-like viruses & Nora & 3 & 0.01 & 1.00
\end{tabular}

322

323 Discussion

324 This study, combined with other recent sequencing efforts, provides new insights into known and previously undescribed viruses associated with Apis Mellifera. Variance analysis revealed a significant, but biologically limited difference in the viral composition between weak and healthy colonies, and no significant difference in the

328 total number of viral sequences derived from healthy and weak colonies could be

329 detected. Genomes from a large number of viral families could be retrieved, of which

330 a substantial part most likely includes plant viruses. While it cannot be excluded that

331 some of the recovered divergent plant viruses constitute viruses actually infecting the

332 bee, it is likely that the majority of these viruses reflect environmental contaminants.

333 The host of the most closely related viral sequence can give an indication if these

334 sequences are environmental contamination. The fact that numerous viral sequences 
belonging to families solely infecting plants were recovered in a large scale viral discovery study in insects indicates that this assumption does not necessarily hold true (47). The recent detection of viruses belonging to plant-specific viral families in mosquitoes reinforces this observation (48). Some of the retrieved viral sequences were very similar to recently discovered viruses (Apis Rhabdovirus 1, Apis

340 Orthomyxovirus 1), increasing the likelihood that these are true honey bee viruses, and further confirming their presence in Belgium. Interestingly, a divergent recombinant Lake Sinai virus was found, comprised of a Hepe-like polymerase region, and a divergent Lake Sinai virus capsid. A novel divergent rhabdovirus (Apis Rhabdovirus 3) was also described. Additionally, full genomes for an orthomyxovirus (Apis Orthomyxovirus 1), very similar to a virus from a previous study from Levin et al. (35), was found in multiple individual libraries, and evidence for the presence of this virus was found in other Hymenoptera families (Table 1). Multiple sequence alignment-free network analysis implied that, despite the species accumulation curves reaching a plateau, many of the putative viral sequences retrieved were too

350 fragmented to be included in a phylogenetic analysis (the number of sequences that made the threshold to be included in phylogenies was 188 , while the network reflected 5,224 retrieved sequences). Furthermore, this analysis implies that the actual viral diversity exceeds what can be captured by regular phylogenetic analysis. Larger sample sizes and especially deeper sequencing efforts could help to fully elucidate the viral diversity associated with honey bees. Since the retrieved non-redundant viral sequence set encapsulates nearly all of the known and even more recently described viruses, this set was used to probe pre-existing Hymenopteran sequencing datasets for any bee-related viral signal. A total of 1,331 virus-rich RNA sequencing datasets were de novo assembled and screened for viruses. This approach revealed that these 
360 datasets harbor a substantial number of viruses that have been previously described

361 (roughly 40\%), but also that the amount of undescribed, divergent viruses is rampant.

362 In concordance with the previous results, the viral sequences retrieved from the SRA

363 search also suffer from fragmentation and incomplete sequencing. This observation is

364 most likely the result of the fact that most RNA sequencing datasets included in the

365 SRA search are transcriptome studies rather than metagenomic analyses, and that for most of them no wet-lab procedures for microbial or viral enrichment were performed.

367 Despite this setback, multiple-sequence alignment free network analysis implied a massive hidden viral diversity within the Hymenoptera lineage (roughly $60 \%$ of the

369 retrieved contigs were less than $90 \%$ similar to any other known virus in Genbank).

370 Constrained ordination analysis showed that both the geographical origin and the

371 taxonomical lineage of the host organism sequenced could explain a biologically relevant proportion (cumulative $\mathrm{R}_{2}=0.33$ ) of the variance within the viral coverage matrix. Since the included samples constitute a wide range of taxonomical host

374 lineages, this proportion was below expectations and implies a substantial amount of

375 viral sequences to be shared over eukaryotic Apidae species and Hymenoptera families. This hypothesis was confirmed by cumulative counting of the viral sequences over the different lineages included, based on a rather rigorous coverage threshold for presence/absence. This analysis revealed a non-trivial number of viral sequences, spanning all of the viral orders previously associated with honey bees, being shared across different lineages within the Apidae, but also over other families belonging to the Hymenoptera. Of all the sequences present in the total non-redundant viral dataset, 53\% were shared with another taxonomical lineage (5139 shared sequences, 9655 in total). Since the included SRA dataset suffers strongly from sampling bias, this percentage is most likely an underestimation. Given this strikingly high number of 
385 virus sharing, the dataset was revisited with a subset of previously described honey bee specific viruses. Surprisingly, none of the tested viruses were lineage restricted to

387 Apis Mellifera, with the exception of Apis rhabdovirus. Other viruses, such as Nora388 like viruses, KBV and LSV were restricted to Apis Mellifera within the Apidae lineage

$389(\mathrm{AMSI}=1.00)$ but were underrepresented relative to non-Apidae Hymenopteran

390 families. The only viruses that were bee specific, i.e. having both a high AMSI and

391 ASI, were DWV and Apis rhabdoviruses. These results imply that despite the recent sequencing efforts, many unknowns remain on viral diversity within the Hymenoptera lineage. Finally, the concept of "honey bee specific viruses" should be revisited, since most of the previously described viruses are not bee specific, neither are they restricted to the Apidae lineage.

\section{Methods}

\section{Data and code availability}

399 All relevant (intermediate) output files, metadata tables, fasta sequences, R code,

400 Python code and jupyter notebooks are available on Github through the URL 401 https://github.com/Matthijnssenslab/Bee_euvir. Intermediary output files too large to 402 be hosted on Github are available through Zenodo (10.5281/zenodo.3979324). The raw sequencing data is available through the SRA database under project accession PRJNA579886. Accession numbers of the viral sequences included in the phylogenies will be made available in supplemental table S1. Accession information for the public datasets screened in this study are available in supplemental table S2. 


\section{Sample preparation, pooling, VLP-sequencing and read processing}

409 Samples were pooled and prepared for Illumina sequencing as described before, and

410 the prokaryotic viruses in these pools were described previously (42). Briefly, samples

411 were taken from the Flanders EpiloBEE study (40), from both sampling years (2012

412 and 2013), and 102 pools were constructed based on health status (defined

413 retrospectively within the EpiloBEE study, with "strong" hives surviving winter and

414 "weak" hives not surviving winter), subspecies and geographical location. Pooling

415 information and SRA accession numbers were described before (42). After

416 sequencing, reads were quality controlled using Trimmomatic (49), version 0.38.

417 Subsequently, de novo assemblies were made for the individual libraries using 418 SPAdes (50), version 3.12.0, with kmer sizes 21, 33, 55 and 77 in the metagenomic

419 mode. To remove redundancy, the resulting contigs larger than 500 bp were collapsed

420 if they showed $97 \%$ nucleotide identity over at least $80 \%$ of the contig lengths, using

421 ClusterGenomes (https://bitbucket.org/MAVERICLab/docker-clustergenomes).

422 Putative eukaryotic viruses were identified using the BlastX method implemented in

423 DIAMOND (43) version 0.9.22, using the 'c 1' and 'sensitive' flags, against the NR database (NCBI), downloaded on 30 september 2018. Taxonomical paths were parsed with the KtClassifyBLAST algorithm implemented in Kronatools (44). All contigs that fell under taxID '10239' (Viruses) were included in the analysis. Contigs

427 that could be annotated as bacteriophages (as described before (42)) were excluded.

428 Coverage values per sample were obtained by mapping the reads per sample back to 429 the viral dataset, using BWA-mem version 0.7.16a (51), filtering the obtained 430 alignments for an identity of $97 \%$ over a coverage of $70 \%$ using BAMM 431 (https://github.com/Ecogenomics/BamM). Coverage values were calculated by 432 dividing the readcounts per contig by the contig length. 


\section{SRA searches}

435 The SRA database was searched by using the query 'Hymenoptera + RNA', and the

436 resulting 5,246 fastQ files were retrieved by using the prefetch and fastq-dump tools

437 implemented in the SRA toolkit (NCBI). The previously obtained viral dataset was used

438 as an index and retrieved fastQ files were mapped back using BWA-mem (51), version

439 0.7.16a. Only samples that had a cumulative read count of at least 100,000 reads

440 (1,331 samples) were included downstream. Samples were then de novo assembled using SKESA (46) and annotated and clustered as described above. Information on the included samples is provided in supplemental table 2.

\section{Phylogenetic analysis}

444 Viral sequences were included based on an ad hoc determined length cut-off 445 depending on the expected genome length of each virus (supplemental table S3).

446 Reference sequences were included by using the retrieved viral sequences as query 447 and performing a TBlastX search (52) with an e-value cutoff of $1 \mathrm{E}-10$ against the nt database (NCBI), downloaded on 1 october 2019. For the Partiti-like, Tymo-like and

449 Toti-like trees only Refseq sequences were included. Significant hits were also filtered 450 on the abovementioned alignment length cut-off specific for a viral group 451 (supplemental table 3). Next, proteins were predicted from both the queries and the significant hits, using prodigal (53), version 2.6.3. Predicted proteins were submitted to an all-to-all BlastP search, with an e-value cut-off of $1 \mathrm{E}-10$. The output was then

454 transformed into a network and the largest connected component was extracted using 455 the networkx library (54) implemented in Python. Proteins within the largest connected component were subsequently aligned with MAFFT (55), version 7.313, using the L- 
458 Model selection was performed using Prottest (57), version 3.4.2. Bayesian

459 phylogenetic analysis was performed using BEAST (45), version 1.10.4, using the

460 predicted protein models (supplemental table 3) under a strict clock and constant

461 population size prior. The respective analysis was ran until all the effective samples

462 sizes were above 200, and maximum clade credibility trees were calculated using

463 TreeAnnotator, implemented in the BEAST package. Final trees were plotted in $\mathrm{R}$ 464 using the ggtree package (58).

$465 \quad$ Network and contig sharing analysis

466 Networks were created from the retrieved viral sequence data by using TBlastX against the Refseq nt database, downloaded on 1 october 2019. The Refseq database was filtered by removing entries containing the keyword 'phage' (for bacteriophages) or 'herpes' in the header, and by removing sequences longer than $15000 \mathrm{nt}$ and shorter than 500 nt. These cut-offs were implemented to reduce 'noisy' hits, where for example herpes polymerases have significant hits to other viral polymerases. The remaining sequences were clustered on $80 \%$ nucleotide identity over $80 \%$ of the length, by using CDhit, version 4.8.1 (59). The tBlastX search was performed with an E-value cutoff of $1 \mathrm{E}-10$ and an alignment length cut-off of 300 positions, and was ran in two iterations to include reference sequences that only made the cut-off when aligning to other reference sequences. The resulting blast output was then converted 477 into a minimized nested block network, using the graph-tool package (60), 478 implemented in Python. Virus sharing over the eukaryotic families belonging to the 479 Hymenoptera and within the Apidae was determined by using the coverage matrix. A 480 viral sequence was assumed to originate from the taxonomical lineage of the sample 481 of which the cluster representative (the longest contig inside a cluster) was derived, in order to determine directionality. A viral representative sequence was assumed to be 
present in a sample when the coverage was above 0.1 . For the cumulative virus sharing, an additional threshold was imposed were at least $10 \%$ of the included samples of a specific taxonomical host lineage had to be positive before the viral sequence was assumed to be present within that lineage. Resulting networks were

487 visualized in Cytoscape (61), version 3.7.1. Percentages of positive samples were calculated using the same relative count cutoff as mentioned before and the ASI and AMSI were calculated by taking the ratio of the fraction of positive samples for a specific bee virus within Apidae or Apis Mellifera samples, divided by the fraction of positive samples in other eukaryotic families or other Apidae species, respectively.

\section{QUANTIFICATION AND STATISTICAL ANALYSIS}

PCoA analysis was performed in $R$ (62) version 3.5.3, with the pcoa function implemented in the 'ape' library (63). Variance analysis and distance-based redundancy analysis was performed on the coverage matrix using Bray-Curtis distances, using the adonis test and capscale function implemented in vegan (64).

497 Cumulative explanation power of the location (country of origin) and eukaryotic Mann-Whitney U test implemented in scipy (65), in Python.

\section{References}

1. vanEngelsdorp D, Meixner MD. A historical review of managed honey bee populations in Europe and the United States and the factors that may affect them. J Invertebr Pathol. 2010 Jan;103:S80-95.

2. Gallai N, Salles J-M, Settele J, Vaissière BE. Economic valuation of the vulnerability of 
4. Aizen MA, Garibaldi LA, Cunningham SA, Klein AM. How much does agriculture depend on pollinators? Lessons from long-term trends in crop production. Ann Bot. 2009 Jun;103(9):1579-88.

5. Hallmann CA, Sorg M, Jongejans E, Siepel H, Hofland N, Schwan H, et al. More than 75 percent decline over 27 years in total flying insect biomass in protected areas. Lamb EG, editor. PLOS ONE. 2017 Oct 18;12(10):e0185809.

6. Potts SG, Biesmeijer JC, Kremen C, Neumann P, Schweiger O, Kunin WE. Global pollinator declines: trends, impacts and drivers. Trends Ecol Evol. 2010 Jun;25(6):34553.

7. Foley JA. Global Consequences of Land Use. Science. 2005 Jul 22;309(5734):570-4.

8. Lusebrink I, Girling RD, Farthing E, Newman TA, Jackson CW, Poppy GM. The Effects of Diesel Exhaust Pollution on Floral Volatiles and the Consequences for Honey Bee Olfaction. J Chem Ecol. 2015 Oct;41(10):904-12.

9. Henry M, Beguin M, Requier F, Rollin O, Odoux J-F, Aupinel P, et al. A Common Pesticide Decreases Foraging Success and Survival in Honey Bees. Science. $2012 \mathrm{Apr}$ 20;336(6079):348-50.

10. Decourtye A, Devillers J, Genecque E, Le Menach K, Budzinski H, Cluzeau S, et al. Comparative sublethal toxicity of nine pesticides on olfactory learning performances of the honeybee Apis mellifera. Arch Environ Contam Toxicol. 2005 Feb;48(2):242-50.

11. Nicholls $\mathrm{Cl}$, Altieri MA. Plant biodiversity enhances bees and other insect pollinators in agroecosystems. A review. Agron Sustain Dev. 2013 Apr;33(2):257-74.

12. Fünfhaus A, Ebeling J, Genersch E. Bacterial pathogens of bees. Curr Opin Insect Sci. 2018 Apr;26:89-96.

13. Forfert N, Natsopoulou ME, Frey E, Rosenkranz P, Paxton RJ, Moritz RFA. Parasites and Pathogens of the Honeybee (Apis mellifera) and Their Influence on Inter-Colonial Transmission. Rueppell O, editor. PLOS ONE. 2015 Oct 9;10(10):e0140337.

14. Grozinger CM, Flenniken ML. Bee Viruses: Ecology, Pathogenicity, and Impacts. Annu Rev Entomol. 2019 Jan 7;64(1):205-26.

15. McMenamin AJ, Flenniken ML. Recently identified bee viruses and their impact on bee pollinators. Curr Opin Insect Sci. 2018 Apr;26:120-9.

16. Engel $P$, Kwong WK, McFrederick $Q$, Anderson KE, Barribeau SM, Chandler JA, et al. The Bee Microbiome: Impact on Bee Health and Model for Evolution and Ecology of HostMicrobe Interactions. mBio [Internet]. 2016 May 4 [cited 2019 Mar 15];7(2). Available from: http://mbio.asm.org/lookup/doi/10.1128/mBio.02164-15

17. Kešnerová L, Emery O, Troilo M, Liberti J, Erkosar B, Engel P. Gut microbiota structure differs between honeybees in winter and summer. ISME J. 2020 Mar;14(3):801-14. 
18. Raymann K, Moran NA. The role of the gut microbiome in health and disease of adult honey bee workers. Curr Opin Insect Sci. 2018 Apr;26:97-104.

19. Leonard SP, Powell JE, Perutka J, Geng P, Heckmann LC, Horak RD, et al. Engineered symbionts activate honey bee immunity and limit pathogens. Science. 2020 Jan 31;367(6477):573-6.

20. Grassl J, Holt S, Cremen N, Peso M, Hahne D, Baer B. Synergistic effects of pathogen and pesticide exposure on honey bee (Apis mellifera) survival and immunity. $J$ Invertebr Pathol. 2018 Nov;159:78-86.

21. Villalobos EM. The mite that jumped, the bee that traveled, the disease that followed. Science. 2016 Feb 5;351(6273):554-6.

22. Annoscia D, Brown SP, Di Prisco G, De Paoli E, Del Fabbro S, Frizzera D, et al. Haemolymph removal by Varroa mite destabilizes the dynamical interaction between immune effectors and virus in bees, as predicted by Volterra's model. Proc R Soc B Biol Sci. 2019 Apr 24;286(1901):20190331.

23. Benaets K, Van Geystelen A, Cardoen D, De Smet L, de Graaf DC, Schoofs L, et al. Covert deformed wing virus infections have long-term deleterious effects on honeybee foraging and survival. Proc R Soc B Biol Sci. 2017 Feb 8;284(1848):20162149.

24. Natsopoulou ME, McMahon DP, Doublet V, Frey E, Rosenkranz P, Paxton RJ. The virulent, emerging genotype $B$ of Deformed wing virus is closely linked to overwinter honeybee worker loss. Sci Rep. 2017 Dec;7(1):5242.

25. Tehel A, Vu Q, Bigot D, Gogol-Döring A, Koch P, Jenkins $C$, et al. The Two Prevalent Genotypes of an Emerging Infectious Disease, Deformed Wing Virus, Cause Equally Low Pupal Mortality and Equally High Wing Deformities in Host Honey Bees. Viruses. 2019 Jan 29;11(2):114.

26. McMenamin AJ, Genersch E. Honey bee colony losses and associated viruses. Curr Opin Insect Sci. 2015 Apr;8:121-9.

27. Alger SA, Burnham PA, Boncristiani HF, Brody AK. RNA virus spillover from managed honeybees (Apis mellifera) to wild bumblebees (Bombus spp.). Rueppell O, editor. PLOS ONE. 2019 Jun 26;14(6):e0217822.

28. Fürst MA, McMahon DP, Osborne JL, Paxton RJ, Brown MJF. Disease associations between honeybees and bumblebees as a threat to wild pollinators. Nature. 2014 Feb;506(7488):364-6.

29. Genersch E, Yue C, Fries I, de Miranda JR. Detection of Deformed wing virus, a honey bee viral pathogen, in bumble bees (Bombus terrestris and Bombus pascuorum) with wing deformities. J Invertebr Pathol. 2006 Jan;91(1):61-3. 
30. Dolezal AG, Hendrix SD, Scavo NA, Carrillo-Tripp J, Harris MA, Wheelock MJ, et al. Honey Bee Viruses in Wild Bees: Viral Prevalence, Loads, and Experimental Inoculation. Rueppell O, editor. PLOS ONE. 2016 Nov 10;11(11):e0166190.

31. Sébastien A, Lester PJ, Hall RJ, Wang J, Moore NE, Gruber MAM. Invasive ants carry novel viruses in their new range and form reservoirs for a honeybee pathogen. Biol Lett. 2015 Sep 30;11(9):20150610.

32. Mordecai GJ, Brettell LE, Pachori P, Villalobos EM, Martin SJ, Jones IM, et al. Moku virus; a new Iflavirus found in wasps, honey bees and Varroa. Sci Rep. 2016 Dec;6(1):34983.

33. Galbraith DA, Fuller ZL, Ray AM, Brockmann A, Frazier M, Gikungu MW, et al. Investigating the viral ecology of global bee communities with high-throughput metagenomics. Sci Rep [Internet]. 2018 Dec [cited 2019 Mar 15];8(1). Available from: http://www.nature.com/articles/s41598-018-27164-z

34. Remnant EJ, Shi M, Buchmann G, Blacquière $T$, Holmes EC, Beekman $M$, et al. A Diverse Range of Novel RNA Viruses in Geographically Distinct Honey Bee Populations. Ross SR, editor. J Virol [Internet]. 2017 Aug 15 [cited 2019 Mar 15];91(16). Available from: http://jvi.asm.org/lookup/doi/10.1128/JVI.00158-17

35. Levin S, Sela N, Erez T, Nestel D, Pettis J, Neumann P, et al. New Viruses from the Ectoparasite Mite Varroa destructor Infesting Apis mellifera and Apis cerana. Viruses. 2019 Jan 24;11(2):94.

36. Daughenbaugh K, Martin M, Brutscher L, Cavigli I, Garcia E, Lavin M, et al. Honey Bee Infecting Lake Sinai Viruses. Viruses. 2015 Jun 23;7(6):3285-309.

37. Schoonvaere K, Smagghe G, Francis F, de Graaf DC. Study of the Metatranscriptome of Eight Social and Solitary Wild Bee Species Reveals Novel Viruses and Bee Parasites. Front Microbiol. 2018 Feb 14;9:177.

38. Gauthier L, Cornman S, Hartmann U, Cousserans F, Evans J, de Miranda J, et al. The Apis mellifera Filamentous Virus Genome. Viruses. 2015 Jul 9;7(7):3798-815.

39. Kraberger S, Cook CN, Schmidlin K, Fontenele RS, Bautista J, Smith B, et al. Diverse single-stranded DNA viruses associated with honey bees (Apis mellifera). Infect Genet Evol. 2019 Jul;71:179-88.

40. Jacques $A$, Laurent $M$, EPILOBEE Consortium, Ribière-Chabert $M$, Saussac $M$, Bougeard $S$, et al. A pan-European epidemiological study reveals honey bee colony survival depends on beekeeper education and disease control. Chaline N, editor. PLOS ONE. 2017 Mar 9;12(3):e0172591.

41. Conceição-Neto N, Zeller M, Lefrère H, De Bruyn P, Beller L, Deboutte W, et al. Modular approach to customise sample preparation procedures for viral metagenomics: a reproducible protocol for virome analysis. Sci Rep [Internet]. 2015 Dec [cited 2018 Oct 4];5(1). Available from: http://www.nature.com/articles/srep16532 
42. Deboutte W, Beller L, Yinda CK, Maes P, de Graaf DC, Matthijnssens J. Honey-beeassociated prokaryotic viral communities reveal wide viral diversity and a profound metabolic coding potential. Proc Natl Acad Sci. 2020 Apr 27;201921859.

43. Buchfink $B, X i e ~ C$, Huson DH. Fast and sensitive protein alignment using DIAMOND. Nat Methods. 2015 Jan;12(1):59-60.

44. Ondov BD, Bergman NH, Phillippy AM. Interactive metagenomic visualization in a Web browser. BMC Bioinformatics. 2011 Dec;12(1):385.

45. Suchard MA, Lemey P, Baele G, Ayres DL, Drummond AJ, Rambaut A. Bayesian phylogenetic and phylodynamic data integration using BEAST 1.10. Virus Evol [Internet]. 2018 Jan 1 [cited 2020 Feb 21];4(1). Available from: https://academic.oup.com/ve/article/doi/10.1093/ve/vey016/5035211

46. Souvorov A, Agarwala R, Lipman DJ. SKESA: strategic k-mer extension for scrupulous assemblies. Genome Biol. 2018 Dec;19(1):153.

47. Shi M, Lin X-D, Tian J-H, Chen L-J, Chen X, Li C-X, et al. Redefining the invertebrate RNA virosphere. Nature. 2016 Dec;540(7634):539-43.

48. Shi C, Beller L, Deboutte W, Yinda KC, Delang L, Vega-Rúa A, et al. Stable distinct core eukaryotic viromes in different mosquito species from Guadeloupe, using single mosquito viral metagenomics. Microbiome. 2019 Dec;7(1):121.

49. Bolger AM, Lohse $M$, Usadel B. Trimmomatic: a flexible trimmer for Illumina sequence data. Bioinformatics. 2014 Aug 1;30(15):2114-20.

50. Bankevich A, Nurk S, Antipov D, Gurevich AA, Dvorkin M, Kulikov AS, et al. SPAdes: A New Genome Assembly Algorithm and Its Applications to Single-Cell Sequencing. J Comput Biol. 2012 May;19(5):455-77.

51. Li H, Durbin R. Fast and accurate short read alignment with Burrows-Wheeler transform. Bioinformatics. 2009 Jul 15;25(14):1754-60.

52. Camacho C, Coulouris G, Avagyan V, Ma N, Papadopoulos J, Bealer K, et al. BLAST+: architecture and applications. BMC Bioinformatics. 2009;10(1):421.

53. Hyatt D, Chen G-L, LoCascio PF, Land ML, Larimer FW, Hauser L. Prodigal: prokaryotic gene recognition and translation initiation site identification. BMC Bioinformatics. 2010 Dec;11(1):119.

54. Hagberg AA, Schult DA, Swart PJ. Exploring Network Structure, Dynamics, and Function using NetworkX. In: Varoquaux G, Vaught T, Millman J, editors. Proceedings of the 7th Python in Science Conference. Pasadena, CA USA; 2008. p. 11-5.

55. Katoh K. MAFFT: a novel method for rapid multiple sequence alignment based on fast Fourier transform. Nucleic Acids Res. 2002 Jul 15;30(14):3059-66. 
56. Capella-Gutierrez S, Silla-Martinez JM, Gabaldon T. trimAl: a tool for automated alignment trimming in large-scale phylogenetic analyses. Bioinformatics. 2009 Aug 1;25(15):1972-3.

57. Darriba D, Taboada GL, Doallo R, Posada D. ProtTest 3: fast selection of best-fit models of protein evolution. Bioinformatics. 2011 Apr 15;27(8):1164-5.

58. Yu G, Lam TT-Y, Zhu H, Guan Y. Two Methods for Mapping and Visualizing Associated Data on Phylogeny Using Ggtree. Battistuzzi FU, editor. Mol Biol Evol. 2018 Dec 1;35(12):3041-3.

59. Fu L, Niu B, Zhu Z, Wu S, Li W. CD-HIT: accelerated for clustering the next-generation sequencing data. Bioinformatics. 2012 Dec;28(23):3150-2.

60. Peixoto TP. The graph-tool python library [Internet]. Figshare; 2017 [cited 2019 Oct 8]. Available from: https://figshare.com/articles/graph_tool/1164194

61. Shannon P. Cytoscape: A Software Environment for Integrated Models of Biomolecular Interaction Networks. Genome Res. 2003 Nov 1;13(11):2498-504.

62. R Core Team. R: A Language and Environment for Statistical Computing [Internet]. Vienna, Austria: R Foundation for Statistical Computing; 2013. Available from: http://www.R-project.org/

63. Paradis E, Schliep K. ape 5.0: an environment for modern phylogenetics and evolutionary analyses in R. Schwartz R, editor. Bioinformatics. 2019 Feb 1;35(3):526-8.

64. Oksanen J, Blanchet FG, Friendly M, Kindt R, Legendre P, McGlinn D, et al. vegan: Community Ecology Package [Internet]. 2019. Available from: https://CRAN.Rproject.org/package=vegan

65. Virtanen P, Gommers R, Oliphant TE, Haberland M, Reddy T, Cournapeau D, et al. SciPy 1.0: Fundamental Algorithms for Scientific Computing in Python. Nat Methods. 2020; 